\title{
Complex Lumbosacral Fracture-Dislocation with Sacral Fracture: Report of a Case and Review of the Literature
}

\section{Liyan Qu}

Zhejiang University

Jiashi Song

Zhejiang University

Fangcai Li

Zhejiang University

Bing Liu ( $\square$ liubingzju@zju.edu.cn )

Zhejiang University

Research article

Keywords: lumbo-iliac fixation, lumbosacral dislocation, sacral fracture

Posted Date: June 29th, 2020

DOI: https://doi.org/10.21203/rs.3.rs-34946/v1

License: (9) This work is licensed under a Creative Commons Attribution 4.0 International License.

Read Full License 


\section{Abstract}

Purpose: Traumatic lumbosacral fracture-dislocation is a very rare pattern of injury. We report a patient who has been suffered from a traffic accident and resulted in concomitant severe injuries: complex lumbosacral fracture-dislocation with pelvic ring disruption and vertical shear sacral fracture; Pneumothorax.

Methods: She was transferred to the emergency department at the local provincial hospital and treated with the pelvic external fixation and closed drainage of thoracic cavity. For the further therapy, she was transferred to our hospital. The neurological examination revealed incomplete S1 paraplegia in the right lower extremity, the left lower extremity was normal, and rectal tone was existed. Standard x-rays and computed tomography revealed L5-S1 fracture-dislocation and fractures of the L5 transverse processes. Results: She was treated with S1 laminectomy and L5-S1 interbody fusion using lumbo-iliac internal fixation.

Conclusions: Available literatures on lumbosacral fracture-dislocation were also reviewed which illustrate the pathogenic, clinical, radiological and therapeutic aspects of lumbosacral fracture dislocation.

\section{Introduction}

Traumatic dislocation of the lumbosacral junction is an unusual injury, characterised by a spondylolisthesis of L5/S1 with or without fractures of the zygapophyseal joints or the pars interarticularis [1]. Sacral fractures can cause pelvic ring instability, but complex lumbosacral fracturedislocation and sacral fracture may result in spinopelvic instability or even dissociation [2]. We present a rare case study of a 34-year-old female who sustained a combination of lumbosacral fracture-dislocation, sacral fracture and pelvic ring injury.

\section{Case Report}

A 34-year-old woman was the victim of a motorcycle accident. The patient was hospitalized and submitted to X-ray examination, revealing the presence of fractures of multiple ribs to the right, pneumothorax, fracture of the right clavicle, dislocated multiple fracture of the pelvic ring (Fig. 1) with fracture-dislocation of L5-S1 and fractures of the right transverse processes of L1-5. In the local provincial hospital, she was treated with the pelvic external fixation (Fig. 2) and closed drainage of thoracic cavity. For the further therapy, she was transferred to our hospital. From the CT scan (Fig. 3), it disclosed fractures of the left L5-S1 facet joint, fractures of the right transverse processes of $L 1, L 2, L 3$, L4 and L5; and the displaced right-sided Zone II longitudinal sacral fracture associated with pelvic ring disruption. The neurological examination revealed incomplete $\$ 1$ paraplegia in the right lower extremity,

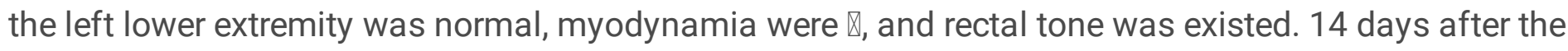
trauma, she was submitted to surgery to treat the vertebral lesion. 
The lumbar and sacral vertebrae were exposed and both were partially resected to allow decompression of the vertebral canal. The S1 nerve root was damaged, but it was still complete. Spinal dura mater was ruptured and resulted in the leakage of cerebrospinal fluid. Pedicle screws were inserted into L5. We can not insert the pedicle screws into S1 for its fracture. Before inserting the iliac screw, the posterior superior iliac spine (PSIS) was exposed and then a $1 \mathrm{~cm} \times 4 \mathrm{~cm}$ bone chip was removed from the inferior part of the PSIS to create a smooth bony bed. A titanium link rod was bent into a desirable profile, attached to the screws, and fastened [3]. The vertical dislocation of the sacrum was reduced by distraction of the rod attached to the lumbar spine. The L5/S1 disc space was fused with a cage containing autologous iliac crest bone graft (Fig. 4). The whole procedure takes $4 \mathrm{~h}$ and the intraoperative bleeding volume was $400 \mathrm{ml}$. In postoperative course, the leakage of cerebrospinal fluid still existed, which was cured by draining and suture. One year later, the lumbosacral and sacral fracture were union, we removed the implant (Fig. 5). On her seven years follow-up, the patient was asymptomatic, pain free, and was able to return to her prior active lifestyle.

\section{Discussion}

Traumatic dislocation of the lumbosacral junction was first described by Watson-Jones in 1940[4]. Traumatic lumbosacral dislocation is a rare lesion often characterised by a fracture dislocation of L5-S1 articular facets associated with anterior L5 slipping. The lesion may be partial or complete and one or both articular processes may be affected. The combined injury of lumbosacral fracture-dislocation, sacral fracture and pelvic ring injury is rare.

\section{Anatomy}

The sacrum is a kyphotic structure with a sagittal angulation from $0^{\circ}$ to $90^{\circ}$, this contributes to the sacral inclination angle of the superior end plate of $\mathrm{S} 1$, which determines the compensatory lordosis of the lumbar spine. The sacrum articulates with four bones: the last lumbar vertebra above via a disc space and facet joint complex, the coccyx below with a ligamentous attachment and occasional bone union, and on either side with the ilium via the sacroiliac joint [5].

\section{Classification}

Several classification systems for sacral fractures have been developed by Tile [6], Denis et al. [7], RoyCamille et al. [8], Strange-Vognsen and Lebech[9] and Isler[10, 11].

Vialle et al[12] reviewed 11 cases and proposed a new anatomical classification of traumatic lumbosacral dislocation based on the injury patterns.

Type I represents a pure dislocation of the articular facets without fracture, and can be divided in three subtypes: 
- - IA: unilateral rotary dislocation secondary to rotational traumatic forces.

- - IB: bilateral facet joint dislocation with lateral displacement. Mechanism of injury seems to be hyperflexion associated with a lateral translational force. In this type of injury (lateral subluxation) and even without anterior displacement of the L5 vertebra, severe disc lesions may be found.

- - IC: bilateral dislocation with anterior slippage of the L5 vertebra. The mechanism involves flexion distraction forces.

Type II represents unilateral articular process fracture dislocation. The mechanism is similar to Type IA with higher energy forces producing a fracture of one of the joint facets and a dislocation without fracture on the opposite side. Anterior slippage of L5 is often asymmetrical with associated disc disruption.

Type III represents bilateral fracture dislocation with anterior displacement and disc injury.

- - IIIA are bilateral fracture with anterior displacement of L5 caused mainly by a flexion-distraction mechanism.

- - IIIB are bilateral fracture with rotational displacement associated to anterior slippage of the L5 vertebra. A combination of high-energy forces that includes flexion-extension and lateral or rotational forces is postulated.

Our patient belongs to the Type II, it disclosed that the left L5-S1 facet joint was fracture-dislocation and the right one was integrity, and the displaced right-sided Zone II longitudinal sacral fracture associated with S1 fracture were well visualized.

\section{Clinical Evaluation}

This injury is mostly found in multiple trauma patients, associated pulmonary, abdominal, and vascular and brain injuries, often need immediate surgical and/or anaesthesiological treatment. Meanwhile, the AP pelvis view was difficult to image the upper sacrum; the actual rate of lumbosacral dislocation and fracture of the upper sacrum may be underestimated [13]. Only about $30 \%$ of all sacral fractures can be identified on a single AP pelvis radiograph. So, pelvic inlet and outlet radiographs are recommended [14].

Computed tomography (CT, both spine and pelvis) scan and reconstitution are imperative, which can demonstrate the details of the complex injury and facilitate the classification and operative decisions. MRI can disclose the neural compression and displacement of the fractures.

This case suffered a high-energy injury, which resulted in the multiple injuries: fractures of multiple ribs to the right, pneumothorax, fracture of the right clavicle, dislocated multiple fracture of the pelvic ring with fracture-dislocation of L5-S1 and fractures of the right transverse processes of L1-5. She was treated according to the Advanced Trauma Life Support protocol and the principles of damage control orthopaedics (immediate temporary stabilisation and secondary definite treatment) [15]. In the local 
provincial hospital, she was treated with the pelvic external fixation and closed drainage of thoracic cavity.

\section{Surgical Operation}

It is usually the result of a high-energy injury with a high incidence of neurological injuries and continue to pose diagnostic and treatment challenges. In this case, although it was severe fracture-dislocation of L5S1, the bilateral pedicles of L5 were not damaged, so the insertion of a pedicle screw at L5 was available. Because of the fracture of S1, the insertion of a pedicle screw at S1 was quite difficult, and then the iliac fixation was undergone. Decompression surgery was performed directly with laminectomy or focal foraminotomy of L5-S1 a before the reduction, thereby preventing stretching of nerve roots and related complications. Caudal screws are implanted into the iliac bone through the posterior-superior-iliac-spine (PSIS) and parallel to the sacroiliac joint $[11,13]$. The two-point iliac fixation creates excellent rotational stability for maintaining correct alignment [16]. The vertical dislocation of the sacrum was reduced by distraction of the rod attached to the lumbar spine. The L5/S1 disc space was replaced with a cage containing autologous iliac crest bone graft. The segmental lumbopelvic fixation system was CD Horizon Legacy, produced by Medtronic Sofamor Danek, USA. The Surgical operation was similar to Liu HC et al [3] and $\mathrm{Yi} C$ et al [11]. To enable reduction, a resection of the facets can be helpful. To achieve a fusion a PLIF procedure or an ALIF procedure combined with posterior instrumentation is possible [1].

\section{Conclusion}

Complex lumbosacral fracture-dislocation with pelvic ring disruption and sacral fracture is a rare lesion. Most authors consider a combination of hyperflexion with compression as the commonest mechanism of injury to produce bilateral L5-S1 dislocation [17-19]. Such as jump from height in a suicidal attempt, other mechanisms of injury include falls from height, traffic accidents and crush injuries. CT scans are essential to detect the whole extent of the lesion. Dural tears should be closed, torn discs should be removed and an anterior interbody fusion by PLIF as a primary option or ALIF as second stage procedure should be performed. Decompression, L5-S1 fusion and lumbo-iliac internal fixation can attain high instability both in lumbosacral alignment and pelvic ring, which may benefit the patients who had complex lumbosacral fracture-dislocation with pelvic ring disruption and sacral fracture.

\section{Declarations}

\section{Ethics approval and consent to participate}

This research has been performed with the approval of an appropriate ethics committee, which is in compliance with the Helsinki Declaration. We confirm that the patient has given their consent for the case report.

\section{Consent for publication}


All of authors consent for publication.

\section{Availability of data and materials}

All of data and materials are available.

\section{Competing interests}

None declared.

\section{Funding}

This research was supported by Grants from the National Natural Science Foundation of China (NO. 81872181).

\section{Authors' contributions}

Bing Liu and Fangcai Li:operation, manuscript.

Liyan Qu: manuscript,literature.

Jiashi Song:fellow up.

\section{Acknowledgements}

No.

\section{References}

1.Schmid R, Reinhold M, Blauth M. (2010)Lumbosacral dislocation: a review of the literature and current aspects of management. Injury. 41:321-8.

2.Schildhauer TA, Bellabarba C, Nork SE, et al.( 2006) Decompression and lumbopelvic fixation for sacral fracture-dislocations with spino-pelvic dissociation. J Orthop Trauma 20:447-57.

3.Liu HC, Chen YZ, Sang XG, Qi L. (2012) Management of lumbosacropelvic fracture-dislocation using lumbo-iliac internal fixation. Injury. 43:452-7.

4.Watson-Jones, R. (1974)The classic: “Fractures and Joint Injuries” by Sir Reginald Watson-Jones, taken from "Fractures and Joint Injuries," by R. Watson-Jones, Vol. II, 4th ed., Baltimore, Williams and Wilkins Company, 1955. Clin Orthop Relat Res 4-10.

5.Robles LA. (2009)Transverse sacral fractures. Spine J. 9:60-9.

6.Tile M. (1988) Pelvic ring fractures: should they be fixed? J Bone Joint Surg Br 70:1-22. 
7.Denis F, Davis S, Comfort T. (1988) Sacral fractures: an important problem. Retrospective analysis of 236 cases. Clin Orthop Relat Res 227:67-81.

8.Roy-Camille R, Saillant G, Gagna G, et al. (1985) Transverse fracture of the upper sacrum. Suicidal jumper's fracture. Spine 10:838-45.

9.Strange-Vognsen HH, Lebech A. (1991) An unusual type of fracture in the upper sacrum. J Orthop Trauma;5:200-3.

10.Isler B. (1990) Lumbosacral lesions associated with pelvic ring injuries. J Orthop Trauma 4:1-6.

11.Yi C, Hak DJ. (2012)Traumatic spinopelvic dissociation or U-shaped sacral fracture: A review of the literature. Injury. 43:402-8.

12.Vialle R, Charosky S, Rillardon L, et al. (2007) Traumatic dislocation of the lumbosacral junction diagnosis, anatomical classification and surgical strategy. Injury 38:169-81.

13.Roche PH, Dufour H, Graziani N, et al. (1998) Anterior lumbosacral dislocation: case report and review of the literature. Surg Neurol 50:11-6.

14.Routt Jr ML, Simonian PT, Swiontkowski MF. (1997)Stabilization of pelvic ring disruptions. Orthop Clin North Am 28:369-88.

15.Pape H-C, Giannoudis P, Krettek C. (2002) The timing of fracture treatment in polytrauma patients: relevance of damage control orthopaedic surgery. Am J Surg 183:622-9.

16.Sar C, Kilicoglu O. (2003)S1 pediculoiliac screw fixation in instabilities of the sacroiliac complex: biomechanical study and report of two cases. J Orthop Trauma 17:262-70.

17.Das De S, McCreath SW. (1981)Lumbosacral fracture-dislocations. A report of four cases. J Bone Joint Surg [Br] 63-B:58-60.

18.Samberg LC. (1975) Fracture-dislocation of the lumbosacral spine. A case report. J Bone Joint Surg [Am] 57:1007-8.

19.Wilchinsky ME. (1987) Traumatic lumbosacral dislocation. A case report and review of the literature. Orthopedics 10:1271-4.

\section{Figures}




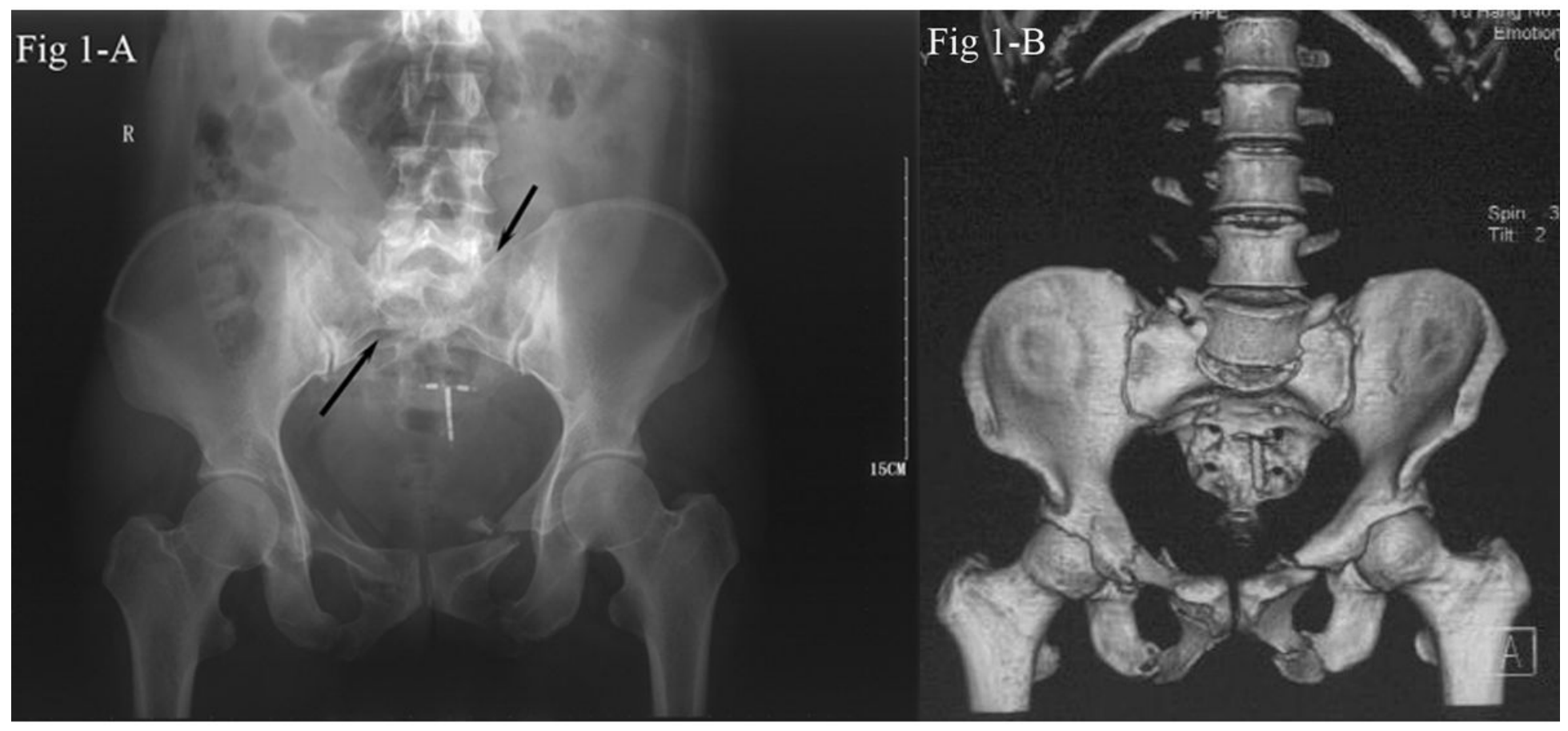

\section{Figure 1}

A radiographic study (Fig.A) and 3-dimensional CT scans (Fig.B) showed fracture of the left L5-S1 facet joint, fractures of the right transverse processes of $L 1, L 2, L 3, L 4$ and $L 5$; and the displaced right-sided Zone II longitudinal sacral fracture associated with pelvic ring disruption.

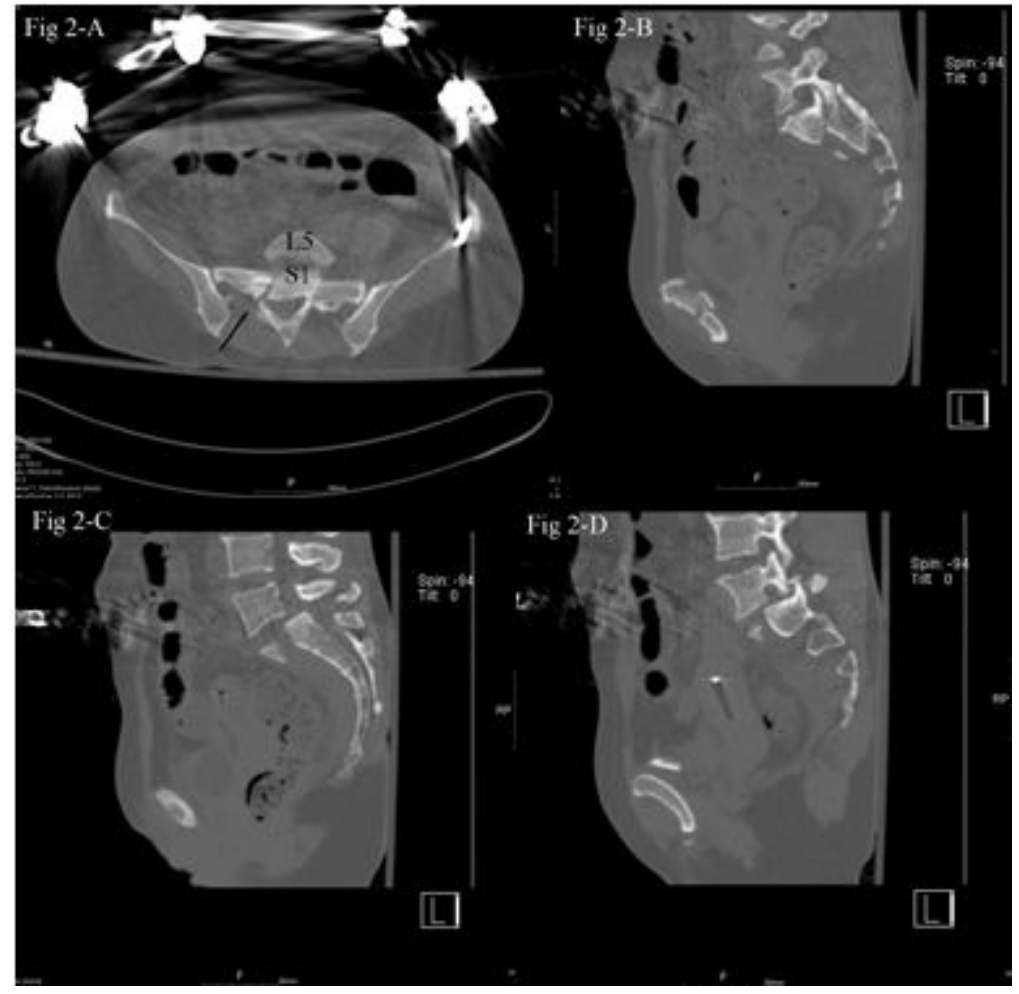

Figure 2 
Preoperative computed tomographic (CT) scans. (A-D) demonstrated left Zone II longitudinal vertical shear sacral fractures, traumatic lumbosacral spondylolisthesis and fracture of the left L5-S1 facet joint.

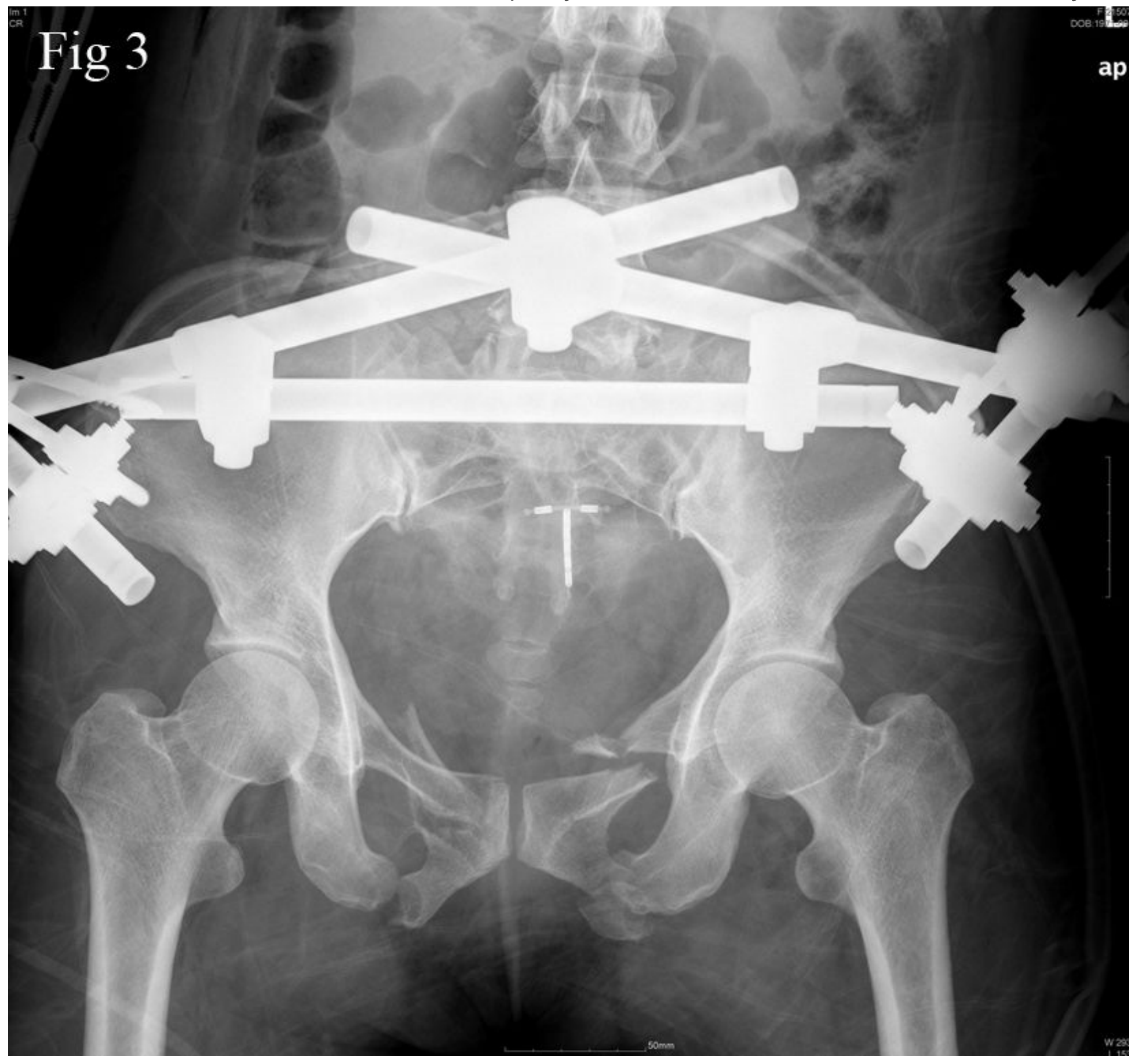

Figure 3

The patient was treated according to the Advanced Trauma Life Support protocol and the principles of damage control orthopaedics. So, she was treated with the pelvic external fixation. 


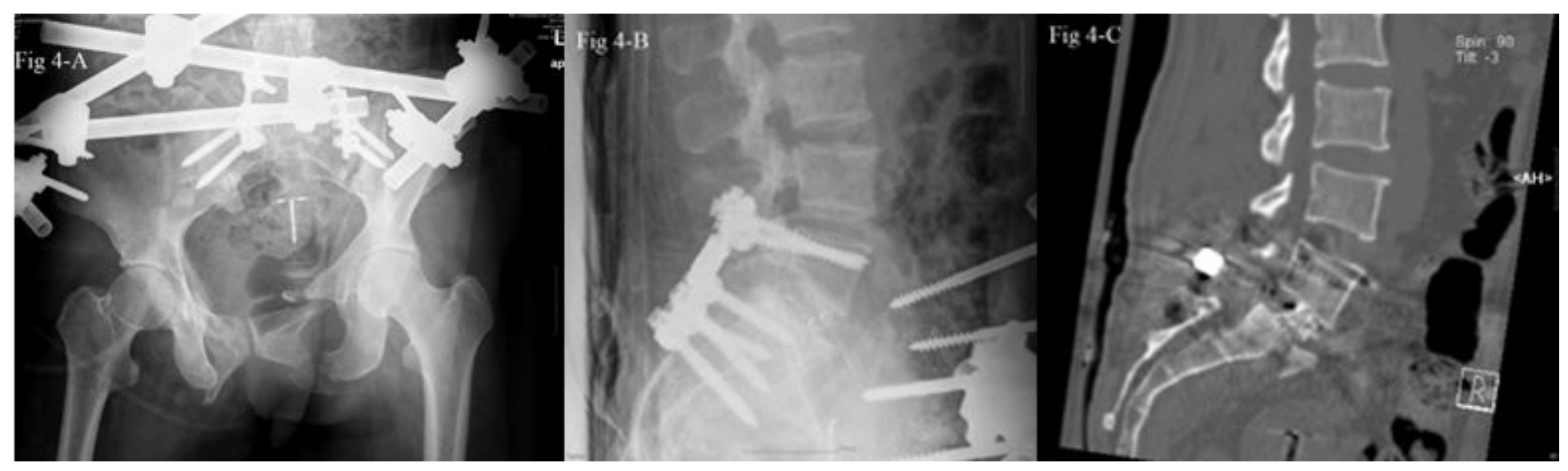

\section{Figure 4}

Postoperative anteroposterior (A), lateral (B) radiographs and CT scan (C) displayed the intact instrumentation of L5 and sacropelvic fixation with iliac wing screws bilaterally. A cage containing autologous iliac crest bone graft can be seen at the L5-S1 level as well. Traumatic lumbosacral spondylolisthesis was reduced.

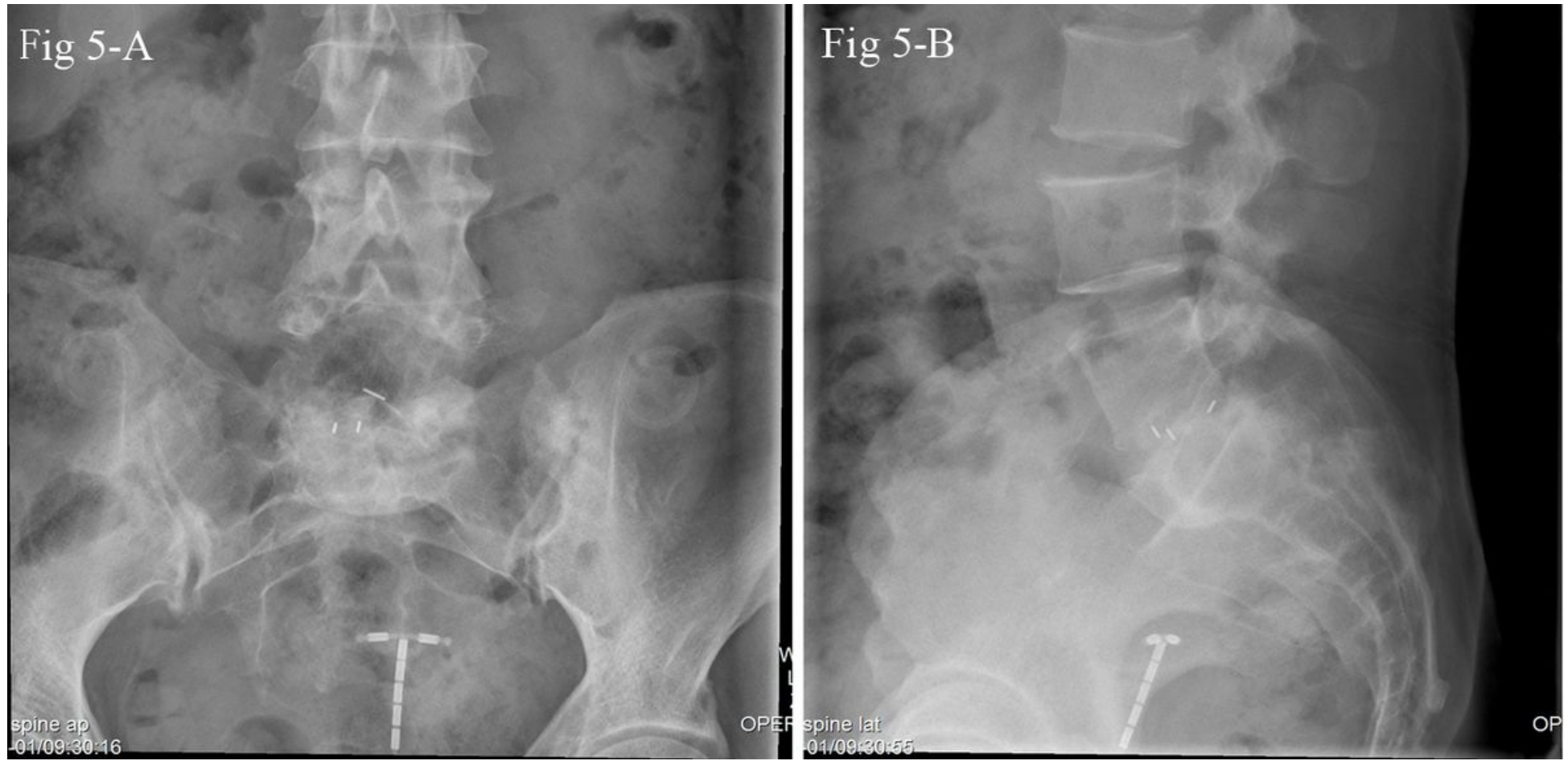

Figure 5

The union of lumbosacral and sacral fracture, and sacropelvic fixation was removed.

\section{Supplementary Files}

This is a list of supplementary files associated with this preprint. Click to download.

- figlegends1.doc 